University of Wollongong

Research Online

Australian Institute for Innovative Materials -

Papers

Australian Institute for Innovative Materials

$1-1-2015$

Hollow carbon spheres with encapsulated germanium as an anode material for lithium ion batteries

\author{
Dan Li \\ University of Wollongong, danli@uow.edu.au \\ Chuanqi Feng \\ Hubei University \\ Hua-Kun Liu \\ University of Wollongong, hua@uow.edu.au \\ Zaiping Guo \\ University of Wollongong, zguo@uow.edu.au
}

Follow this and additional works at: https://ro.uow.edu.au/aiimpapers

Part of the Engineering Commons, and the Physical Sciences and Mathematics Commons

Research Online is the open access institutional repository for the University of Wollongong. For further information contact the UOW Library: research-pubs@uow.edu.au 


\title{
Hollow carbon spheres with encapsulated germanium as an anode material for lithium ion batteries
}

\author{
Abstract \\ A novel composite consisting of hollow carbon spheres with encapsulated germanium (Ge@HCS) was \\ synthesized by introducing a germanium precursor into the porous-structured hollow carbon spheres. The \\ carbon spheres not only function as a scaffold to hold the germanium and thus maintain the structural \\ integrity of the composite, but also increase the electrical conductivity. The voids and vacancies that are \\ formed after the reduction of germanium dioxide to germanium provide free space for accommodating \\ the volume changes during discharging-charging processes, thus preventing pulverization. The obtained \\ Ge@HCS composite exhibits excellent lithium storage performance, as revealed by electrochemical \\ evaluation.

\section{Keywords} \\ carbon, spheres, encapsulated, hollow, germanium, batteries, anode, material, lithium, ion

\section{Disciplines} \\ Engineering | Physical Sciences and Mathematics

\section{Publication Details} \\ Li, D., Feng, C., Liu, H. kun. \& Guo, Z. (2015). Hollow carbon spheres with encapsulated germanium as an \\ anode material for lithium ion batteries. Journal of Materials Chemistry A, 3 (3), 978-981.
}




\section{Hollow Carbon Spheres with Encapsulated Germanium as}

\section{an Anode Material for Lithium Ion Batteries}

Dan $\mathrm{Li}^{\mathrm{a}, \mathrm{b}}$, Chuanqi Feng ${ }^{\mathrm{a}}$, Hua kun Liu ${ }^{\mathrm{b}}$, Zaiping Guo ${ }^{* a, b, c}$

${ }^{a}$ Hubei Collaborative Innovation Center for Advanced Organic Chemical Materials, College of Chemistry and Chemical Engineering, Hubei University, Wuhan 430062, China

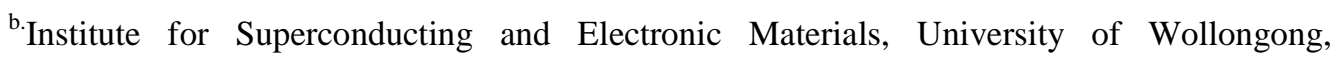
Wollongong, NSW 2522, Australia.

c.School of Mechanical, Materials and Mechatronics Engineering, University of Wollongong, North Wollongong, NSW 2500, Australia

*Corresponding author: E-mail: zguo@uow.edu.au; Tel: +61 242215225 


\begin{abstract}
A novel composite consisting of hollow carbon spheres with encapsulated germanium (Ge@HCS) was synthesized by introducing germanium precursor into the porous-structured hollow carbon spheres. The carbon spheres not only function as a scaffold to hold the germanium and thus maintain the structural integrity of the composite, but also increase the electrical conductivity. The voids and vacancies that are formed after the reduction of germanium dioxide to germanium provide free space for accommodating the volume changes during discharging/charging processes, thus preventing pulverization. The obtained Ge@HCS composite exhibits excellent lithium storage performance, as revealed by electrochemical evaluation.
\end{abstract}

\title{
Keywords
}

Germanium; Hollow carbon sphere; Encapsulation; Anode; Lithium ion battery. 


\section{Introduction}

The increasing demand for clean and green energy is currently shifting towards alternative energy sources such as wind power, tidal power, solar cells, fuel cells, and batteries [1]. Lithium ion batteries have been considered to be the most promising energy storage system for portable electrical devices, electric vehicles, hybrid electric vehicles, etc [2]. Lithium ion batteries are more suitable for such purposes compared to previous conventional batteries, including metal hydride, alkaline, and lead-acid batteries, due to their high power density and high energy density. To further satisfy the demand, much research has been recently focused on high capacity materials. Group IVA materials have attracted significant interest as anode materials for lithium ion batteries due to their high theoretical capacity, especially silicon and germanium, which exhibit high theoretical capacities of 4200 $\mathrm{mA} \mathrm{h} \mathrm{g}{ }^{-1}$ and $1623 \mathrm{~mA} \mathrm{~h} \mathrm{~g}^{-1}$ (4.4 $\mathrm{Li}^{+}$per silicon or germanium atom), respectively [3-5]. In the case of silicon, its practical use in commercial application is limited by inferior cycling performance because of the great volume changes during cycling and the poor rate capability caused by its inherent low ionic diffusivity and electronic conductivity. Compared to silicon, germanium can boost the electrical conductivity to two orders of magnitude higher, and the lithium diffusivity to four orders of magnitude faster $[3,6]$. Furthermore, it has been proved that the total system energy can be dramatically decreased by the high adsorption energy of lithium ions on the germanium surface [5]. These outstanding properties make germanium an excellent candidate anode material for lithium ion batteries. Like silicon, however, the major drawback of germanium is the large volume change during lithiation/de-lithiation processes, which results in cracking, pulverization, and loss of electrode contact, leading to poor cyclability and capacity fading [7]. Synthesis of nanostructured morphologies, such as nanowires [4, 7-9], nanotubes [5, 10], and nanoparticles [11], is an effective method to address this issue by accommodating the volume change, introducing relaxation mechanisms 
to tolerate strain, and alleviating the pulverization. Another significant strategy is developing a carbon-based germanium composite that not only provides a volume expansion buffer for lithium ion intercalation, but also improves the electrical conductivity of the material. There are many previous studies that have reported germanium modified by various carbon sources, for example, nanostructured germanium interconnected by carbon synthesized by a reduction reaction between amorphous $\mathrm{GeO}_{2}$ and carbon [12], single crystalline germanium nanowires sheathed with carbon prepared by a solid-liquid solution method [4], self-assembled germanium-carbon nanostructures obtained through carbon coating and reduction of the oxide precursor [11], carbon covered germanium particles embedded into reduced graphene oxide networks [13], sandwich-structured C/Ge/graphene composite synthesized by a microwaveassisted solvothermal reaction and then carbon-coating the surface of the germanium/graphene precursor [14]. Hollow carbon spheres (HCS) have attracted considerable attention because of their promising applications in catalysis, lithium-ion batteries, fuel cells, supercapacitors, and water treatment [15, 16]. Resorcinol-formaldehyde resin (RF) is considered as one of the most fascinating and versatile carbon precursors for the designed synthesis of HCS [17]. Moreover, it is reported that hexadecyltrimethylammonium bromide (CTAB) modified HCS are endowed with a uniform microporous structure with superior surface properties, which could be beneficial to the introduction of metal precursors. Therefore, their unique structure makes HCS a good candidate for synthesizing a composite with germanium encapsulated in carbon spheres.

Herein, hollow carbon spheres were firstly synthesized using silicon spheres as hard templates. The carbon spheres were modified by CTAB in the process of RF coating to obtain a porous structure, which not only increases the contact area between the electrode and electrolyte, but also facilitates the introduction of the germanium precursor. Within this architecture, the germanium can freely undergo lithiation/de-lithiation processes without agglomeration due to the protecting scaffold effect of the carbon spheres and the voids that 
are formed after the reduction treatment of the precursor, thus securing the structural and electrical integrity of the electrode during repeated cycling. This unique encapsulation of germanium in hollow carbon spheres endows the obtained materials with enhanced electrochemical properties.

\section{Experimental Section}

Synthesis of $\mathrm{SiO}_{2}$ spheres: $\mathrm{SiO}_{2}$ spheres were synthesized by a slightly modified Stöber process [18]. In a typical synthesis, $0.6 \mathrm{~mL}$ tetraethyl orthosilicate (TEOS) was added into a mixture of $63.3 \mathrm{~mL}$ isopropyl alcohol, $23.5 \mathrm{~mL}$ deionized water, and $13.0 \mathrm{~mL}$ ammonium aqueous solution (30\%), and then vigorous stirred at room temperature for $1 \mathrm{~h}$. Then, $5 \mathrm{~mL}$ TEOS was added dropwise into the solution at $35{ }^{\circ} \mathrm{C}$ for $2 \mathrm{~h}$. Then, the $\mathrm{SiO}_{2}$ spheres were centrifugally separated from the suspension and washed with ethanol and deionized water five times before drying in a vacuum oven at $70{ }^{\circ} \mathrm{C}$ overnight.

Synthesis of $\mathrm{SiO}_{2} @ R F$ core-shell spheres: $1 \mathrm{~g}$ of the obtained $\mathrm{SiO}_{2}$ spheres was homogeneously dispersed in a solution of deionized water $(70 \mathrm{~mL})$ and ethanol $(28 \mathrm{~mL})$ by ultrasonication for $30 \mathrm{~min}$, followed by the addition of $2.3 \mathrm{~g}$ hexadecyl trimethyl ammonium bromide (CTAB), $0.35 \mathrm{~g}$ resorcinol, and $0.1 \mathrm{~mL}$ ammonium aqueous solution, and then stirring at room temperature. The mixed solution was homogenized for 30 min to form a uniform dispersion. Then, $0.5 \mathrm{~mL}$ formaldehyde solution was added to the dispersion with continuous stirring for $8 \mathrm{~h}$ at $35{ }^{\circ} \mathrm{C}$. After that, the products, the $\mathrm{SiO}_{2} @ \mathrm{RF}$ core-shell spheres, were collected by centrifugation and washed with ethanol and deionized water five times before drying in a vacuum oven at $70{ }^{\circ} \mathrm{C}$ overnight.

Synthesis of hollow carbon spheres (HCS): The as-prepared $\mathrm{SiO}_{2} @ \mathrm{RF}$ core-shell spheres were carbonized under argon atmosphere at $350{ }^{\circ} \mathrm{C}$ for $1.5 \mathrm{~h}$, followed by a further annealing treatment at $900{ }^{\circ} \mathrm{C}$ for $3 \mathrm{~h}$ with a heating rate of $1{ }^{\circ} \mathrm{C} \mathrm{min}^{-1}$. Finally, the furnace was left to cool down to room temperature before the obtained $\mathrm{SiO}_{2} @ \mathrm{C}$ spheres were removed from the 
tube furnace. After soaking and washing in $3 \mathrm{M} \mathrm{NaOH}$ aqueous solution at $70{ }^{\circ} \mathrm{C}$ for $48 \mathrm{~h}$, the as-synthesized $\mathrm{SiO}_{2} @ \mathrm{C}$ core-shell spheres were converted into HCS.

Synthesis of $\mathrm{GeO}_{2} @$ hollow carbon spheres (GeO $\left.\mathrm{G}_{2} @ \mathrm{HCS}\right): 0.5$ g HCS was sealed in a twoneck flask, which was then subjected to vacuum for $30 \mathrm{~min}$. Then, $1.5 \mathrm{~g}$ germanium ethoxide was injected into the evacuated flask, stirred for $48 \mathrm{~h}$, and washed with ethanol two times to remove extra germanium ethoxide before drying in a vacuum oven at $70{ }^{\circ} \mathrm{C}$ overnight.

Synthesis of Ge@hollow carbon spheres (Ge@HCS): The as-prepared GeO $\mathrm{G}_{2} @ \mathrm{HCS}$ sample was reduced to Ge@HCS under 5\% hydrogen/argon gas at $650{ }^{\circ} \mathrm{C}$ for $10 \mathrm{~h}$ with a heating rate of $5^{\circ} \mathrm{C} \min ^{-1}$.

Synthesis of $\mathrm{GeO}_{2}$ bulks, GeO $\mathrm{O}_{2} @$ carbon bulks (GeO $\mathrm{G}_{2} @ \mathrm{C}$ bulks), and Ge@carbon particles (Ge@C particles): $\mathrm{GeO}_{2}$ bulks were obtained by the natural hydrolysis of germanium ethoxide in a sealed vial for $48 \mathrm{~h}$. Then, the obtained $\mathrm{GeO}_{2}$ bulks were coated with a carbon layer through carbonization in ethanol vapour in nitrogen at $650{ }^{\circ} \mathrm{C}$ for $3 \mathrm{~h}$ with a heating rate of $5{ }^{\circ} \mathrm{C}$ min $^{-1}$ to obtain the $\mathrm{GeO}_{2} @ \mathrm{C}$ bulks. The Ge@C particles were synthesized by reducing the as-prepared $\mathrm{GeO}_{2} @ \mathrm{C}$ bulks under 5\% hydrogen/argon gas at $650{ }^{\circ} \mathrm{C}$ for $10 \mathrm{~h}$ with a heating rate of $5^{\circ} \mathrm{C} \min ^{-1}$.

Characterization: The crystalline phases of the resultant materials were analysed by powder X-ray diffraction (XRD, MMA GBC, Australia), which was carried out using $\mathrm{Cu}$ K $\alpha$ radiation $(\lambda=1.54056 \AA)$ from $2 \theta .=10^{\circ}$ to $80^{\circ}$. The morphologies and structures of the samples were characterized by field-emission scanning electron microscopy (SEM, JSM-7500FA, JEOL, Japan), and transmission electron microscopy (TEM) investigations were performed using a JEOL 2011F analytical electron microscope (JEOL, Japan) operating at $200 \mathrm{keV}$. Raman spectroscopy was used to characterize the germanium and carbon using $514 \mathrm{~nm}$ laser excitation. Thermogravimetric analysis (TGA) was carried out to determine the carbon content with a TGA/differential scanning calorimetry (DSC) type instrument (METTLER 
TOLEDO, Switzerland) at a heating rate of $10{ }^{\circ} \mathrm{C} \min ^{-1}$ from room temperature to $800{ }^{\circ} \mathrm{C}$ in air.

Electrochemical Measurements: To prepare the working electrode for electrochemical testing, an electrode slurry of Ge@HCS was prepared by thoroughly mixing the active material, polyacrylic acid, sodium carboxymethyl cellulose, and acetylene black in a weight ratio of 80:5:5:10 in de-ionized water. For the Ge@C particle sample, the slurry was made up in a weight ratio of 83.2:4.2:4.2:8.4 in order to keep the same carbon content as in the Ge@HCS electrode. The resultant slurries were then spread onto copper foil substrates and dried in a vacuum oven at $150{ }^{\circ} \mathrm{C}$ for $3 \mathrm{~h}$. The electrochemical tests were carried out with CR2032 coin type cells, which were assembled in an argon-filled golve box(Mbraun, Unilab, Germany). The cells were constructed of the prepared active material on copper substrate as cathode, microporous polyethylene (Celgard 2400) as the separator, lithium foil as anode, and $1.15 \mathrm{M}$ $\mathrm{LiPF}_{6}$ in a ethylene carbonate (EC)/dimethyl carbonate (DMC)/diethyl carbonate (DEC) (3:4:3 by volume) as the electrolyte. The charge/discharge cycling was performed within the voltage range of 0.01-1.5 V vs. $\mathrm{Li}^{+} / \mathrm{Li}$ on a battery test instrument (CT2001A, KINGNUO, China) at ambient temperature. The loading amount of active material for all electrodes was at least $0.9 \mathrm{mg} \mathrm{cm}^{-2}$. The specific capacities were calculated based on the weight of the composites.

\section{Results and Discussion}

Figure 1 illustrates the general route to synthesize the Ge@HCS composite through introducing germanium ethoxide into the hollow carbon spheres, followed by a thermal reduction treatment. In a typical synthetic procedure, the $\mathrm{SiO}_{2}$ template spheres are synthesized by a modified Stöber method and then covered with a uniformed carbon layer transformed from carbonization of the self-assembled RF and CTAB. After etching with 
cationic surfactant CTAB plays a significant role in the surface properties of the silica templates. The cationic CTAB can decorate the spheres with positive charges by forming a bilayer structure, which can easily make the negatively charged RF form a coating on the negatively charged Stöber silica spheres [19], promoting the following self-assembly of CTAB and RF on the silica sphere surfaces to form a polymer shell. Importantly, the CTAB modified polymer shells can be transformed to uniform microporous carbon spheres because of the elimination of CTAB after the carbonization treatment [20]. Therefore, the structure features carbon spheres equipped with high dispersibility and discreteness [21], which can have an affinity with the germanium ethoxide and thus allow it to penetrate the carbon layer and go into the center of a hollow carbon sphere under vacuum conditions. After a long stirring over 2 days, the vacuum beaker is gradually filled with air to ensure that the germanium ethoxide is hydrolyzed to $\mathrm{Ge}(\mathrm{OH})_{4}$ and then converted to $\mathrm{GeO}_{2}$. The obtained $\mathrm{GeO}_{2} @ \mathrm{HCS}$ undergoes a thermal reduction treatment and is finally transformed to Ge@HCS.

Figure S1 and Figure 1(a) contain SEM images of the $\mathrm{SiO}_{2}$ templates, which consist of relatively monodisperse nanospheres about $250 \mathrm{~nm}$ in size. After the coating with RF resin and the following carbonization treatment, the hollow carbon spheres were obtained, which kept their uniform and monodisperse structure, as can be observed in the SEM and TEM images in Figure S2. Germanium ethoxide was introduced into the carbon spheres under vacuum and converted to $\mathrm{GeO}_{2}$ after a long stirring process to facilitate a complete hydrolysis and oxidation reaction. The vacuum strategy is effective for loading the germanium precursor, which can be proved from the TEM image shown in Figure 2(b), indicating that the hollow centers of the HCS were fully occupied by $\mathrm{GeO}_{2}$. SEM images of $\mathrm{GeO}_{2} @ \mathrm{HCS}$ are shown in Figure S3. After completion of the thermal reduction process, relatively thinner areas appeared inside the carbon spheres, which were stems from the produced voids and pores due to the release of oxygen when $\mathrm{GeO}_{2}$ was reduced to elemental germanium (See Figure 2(c)). 
From the SEM images (as shown in Figure S4), it can be found from broken carbon spheres that there are germanium particles inside the carbon spheres. Energy dispersive $\mathrm{X}$-ray spectroscopy (EDS) gives further evidence to confirm the presence of germanium in the Ge@HCS sample in Figure S5. On the other hand, $\mathrm{GeO}_{2} @ \mathrm{C}$ particles were synthesized for comparison by natural hydrolysis of the germanium ethoxide precursor without the presence of HCS under the identical synthesis conditions as those for the $\mathrm{GeO}_{2} @ \mathrm{HCS}$ sample. The asprepared $\mathrm{GeO}_{2}$ bulks (shown in Figure S6) were transformed to $\mathrm{GeO}_{2} / \mathrm{C}$ bulks after the carbon-coating treatment, and then were further transformed to Ge@C particles after the thermal reduction process. Images of the morphology of the $\mathrm{GeO}_{2} / \mathrm{C}$ bulks and $\mathrm{Ge} @ \mathrm{C}$ particles are shown in Figures S7 and S8, respectively. X-ray diffraction (XRD) was applied to characterize the crystal structures of the obtained $\mathrm{GeO}_{2} @ \mathrm{HCS}$ and Ge@HCS samples, as shown in Figure 2(d). All the reflection peaks of the $\mathrm{GeO}_{2} @ \mathrm{HCS}$ are well indexed to the hexagonal phase of $\mathrm{GeO}_{2}$ (JCPDS card No. 36-1463). The hexagonal phase is converted to diamond cubic phase (Ge) (JCPDS card No. 40-0545) after the reduction treatment. No peaks corresponding to carbon were detected in the pattern, which could be attributed to their overlapping with the (111) peak of germanium at around $27^{\circ}$. Further structural information is provided by the Raman spectra, as shown in Figure S9. The peak located at $294 \mathrm{~cm}^{-1}$ is attributed to the optical mode of crystalline germanium. Moreover, there is a strong D band at $1345 \mathrm{~cm}^{-1}$ indicating the presence of defects, and a G band at $1605 \mathrm{~cm}^{-1}$ that is attributable to the $E_{2 \mathrm{~g}}$ vibrational mode for the $s p^{2}$ domain [22]. The carbon content of the Ge@C particles and Ge@HCS composites was determined by thermogravimetric analysis (TGA), with the results shown in Figure S10. The calculated values are $11.9 \mathrm{wt} \%$ and $10.3 \mathrm{wt} \%$, respectively.

The hollow carbon spheres encapsulating germanium with structural stability can offer Comment [HL3]: Add the explaination of different behaviors of two curves for S10. Why the Ge@C is oxidized and Ge@HCS is not oxidized after $600^{\circ} \mathrm{C}$ ? excellent lithium storage performance, and the results are shown in Figure 3. For comparison, the cycling performances of Ge@C particles and Ge@HCS electrode are exhibited in Figure 
3(a) up to 100 cycles at the rate of $0.4 \mathrm{C}$. The current density for the $1 \mathrm{C}$ rate is $1600 \mathrm{~mA} \mathrm{~g}^{-1}$. The Ge@HCS electrode delivers a significantly high specific capacity of about 1455 mA h g ${ }^{-1}$ at the first cycle, corresponding to the quite high specific capacity of $1617 \mathrm{~mA} \mathrm{~h} \mathrm{~g}^{-1}$ for germanium alone, which is very close to the theoretical capacity of germanium. The capacity remains almost constant after the fifth cycle. On the o`ther hand, it can be observed that the Comment [HL4]: 'other' Ge@C particle electrode shows very fast capacity fading with only a negligible specific capacity value of $185 \mathrm{~mA} \mathrm{~h} \mathrm{~g}^{-1}$ at the $100^{\text {th }}$ cycle. The rate capability of the Ge@HCS electrode was evaluated at various current rates, and the obtained results are shown in Figure 3(b) (charging at 0.1 - $20 \mathrm{C}$ and discharging at $0.2 \mathrm{C}$ ). The Ge@HCS shows much enhanced rate performance compared to the Ge@C particle sample at all current rates. There was a negligible capacity drop when the charging current was increased from $1 \mathrm{C}$ to $3 \mathrm{C}$. The discharge capacity was $1153.3 \mathrm{~mA} \mathrm{~h} \mathrm{~g}^{-1}$ at the $10^{\text {th }}$ cycle at the rate of $0.1 \mathrm{C}, 953 \mathrm{~mA} \mathrm{~h} \mathrm{~g}^{-1}$ at the $50^{\text {th }}$ cycle at $5 \mathrm{C}$, and $878.9 \mathrm{~mA} \mathrm{~h} \mathrm{~g}^{-1}$ at the $60^{\text {th }}$ cycle at the rate of $10 \mathrm{C}$. Even at the high rate of $20 \mathrm{C}$, the specific capacity remained $772.5 \mathrm{~mA} \mathrm{~h} \mathrm{~g}^{-1}$, which is still $67 \%$ of its capacity at $0.1 \mathrm{C}$. Importantly, the specific capacity reached a value of $1140 \mathrm{~mA} \mathrm{~h} \mathrm{~g}^{-1}$ after the high rate measurement and therefore almost recovered, implying their good reversibility. In contrast, an extremely low capacity of only 20 mA h g${ }^{-1}$ was obtained from the Ge@C particle sample at the rate of 20 C. The fast capacity fading and poor capacity recovery of the Ge@C particles are probably ascribe to the pulverization of germanium particles induced by the unavoidable volume changes during the alloying/de-alloying processes, leading to the loss of electrical contact. Figure 3(c) shows the corresponding galvanostatic voltage profiles of the Ge@HCS composite electrode in the $1^{\text {st }}, 2^{\text {nd }}, 12^{\text {nd }}, 22^{\text {nd }}, 32^{\text {nd }}, 42^{\text {nd }}, 52^{\text {nd }}$, and $62^{\text {nd }}$ cycles at different rates, showing that all lithium ion insertion and extraction processes are spread over a relatively broad voltage range, and therefore, are also separated temporally. 
The much improved cycling stability and rate capability of the Ge@HCS sample may be ascribed to the distinct structure and morphology, which offer the following benefits. Firstly, the hollow carbon spheres can function as a physical matrix to effectively prevent the germanium cores from coalescing into a bulk during lithiation/de-lithiation processes, and also protect the germanium cores against pulverization. Secondly, the germanium cores are interconnected by the shells of the carbon spheres, forming an effective and continuous conductive network to significantly facilitate the diffusion and transport of lithium ions even at high current rates. Thirdly, the inherently porous nature of Ge@HCS with many voids can ensure a high contact area between the electrolyte and electrode materials, and thus facilitate the transport and diffusion of lithium ions. More importantly, the porous property can endow the composite with free space to accommodate the volume changes in the germanium during the lithium ion insertion/extraction processes, thus maintaining the structural integrity of the electrode.

\section{Conclusions}

In summary, a novel composite consisting of hollow carbon spheres with encapsulated germanium was first synthesized through infiltrating the germanium precursor into the carbon spheres, which were obtained by carbonization of a CTAB modified RF resin coating on silicon template spheres, followed by etching the templates. The cationic surfactant CTAB served as a soft template for mesostructured RF resin formation, resulting in a porous carbon shell, which facilitates the access of the germanium precursor to its hollow center. The obtained Ge@HCS material shows excellent electrochemical properties, which can be attributed to the unique morphology and structure. The voids and vacancies that are formed after the shrinkage of $\mathrm{GeO}_{2}$ to form germanium can effective accommodate the volume changes during the lithiation/de-lithiation processes, enabling structural integrity and continuity. Meanwhile, the carbon spheres can serve as a network of electronic pathways, 
which could increase the kinetics of lithium reactions. The strategy of encapsulating germanium in HCS could offer a new method for developing anode electrodes with high performance.

\section{Acknowledgements}

This work was partially supported by the National Natural Science Foundation of China (21476063), and the Australian Research Council through a Discovery Project (DP1094261). Part of this research was undertaken at Prof. Xiongwen Lou's laboratory at Nanyang Technological University, and the authors acknowledge the great support and help that they received from Prof. Xiongwen Lou and Dr. Xinyao Yu. The authors also would like to thank Dr. Tania Silver for critical reading of the manuscript. 


\section{References}

[1] C.-M. Park, J.-H. Kim, H. Kim, H.-J. Sohn, Chem. Soc. Rev. 39 (2010) 3115-3141.

[2] L. Ji, Z. Lin, M. Alcoutlabi, X. Zhang, Energy Environ. Sci. 4 (2011) 2682-2699.

[3] H. Lee, J. Cho, Nano Lett. 7 (2007) 2638-2641.

[4] M.-H. Seo, M. Park, K. T. Lee, K. Kim, J. Kim, J. Cho, Energy Environ. Sci. 4 (2011) 425-428.

[5] T. Song, H. Cheng, H. Choi, J.-H. Lee, H. Han, D. H. Lee, D. S. Yoo, M.-S. Kwon, J.M. Choi, S. G. Doo, H. Chang, J. Xiao, Y. Huang, W. I. Park, Y.-C. Chung, H. Kim, J. A. Rogers, U. Paik, ACS Nano 6 (2011) 303-309.

[6] J. Graetz, C. C. Ahn, R. Yazami, B. Fultz, J. Electrochem. Soc. 151 (2004) A698A702.

[7] F.-W. Yuan, H.-J. Yang, H.-Y. Tuan, ACS Nano 6 (2012) 9932-9942.

[8] C. K. Chan, X. F. Zhang, Y. Cui, Nano Lett. 8 (2007) 307-309.

[9] X. H. Liu, S. Huang, S. T. Picraux, J. Li, T. Zhu, J. Y. Huang, Nano Lett. 11 (2011) 3991-3997.

[10] M.-H. Park, Y. Cho, K. Kim, J. Kim, M. Liu, J. Cho, Angew. Chem., Int. Ed. 50 (2011) 9647-9650.

[11] K. H. Seng, M.-H. Park, Z. P. Guo, H. K. Liu, J. Cho, Angew. Chem., Int. Ed. 51 (2012) 5657-5661.

[12] D. T. Ngo, R. S. Kalubarme, H. T. T. Le, J. G. Fisher, C.-N. Park, I.-D. Kim, C.-J. Park, Adv. Funct. Mater. 24 (2014) 5291-5298.

[13] D.-J. Xue, S. Xin, Y. Yan, K.-C. Jiang, Y.-X. Yin, Y.-G. Guo, L.-J. Wan, J. Am. Chem. Soc. 134 (2012) 2512-2515.

[14] D. Li, K. H. Seng, D. Shi, Z. Chen, H. K. Liu, Z. Guo, J. Mater. Chem. A 1 (2013) $14115-14121$. 
[15] K. T. Lee, Y. S. Jung, S. M. Oh, J. Am. Chem. Soc. 125 (2003) 5652-5653.

[16] W.-M. Zhang, J.-S. Hu, Y.-G. Guo, S.-F. Zheng, L.-S. Zhong, W.-G. Song, L.-J. Wan, Adv. Mater. 20 (2008) 1160-1165.

[17] F. Zhang, Y. Meng, D. Gu, Yan, C. Yu, B. Tu, D. Zhao, J. Am. Chem. Soc. 127 (2005) 13508-13509.

[18] B. Guan, X. Wang, Y. Xiao, Y. Liu, Q. Huo, Nanoscale 5 (2013) 2469-2475.

[19] N. Li, Q. Zhang, J. Liu, J. Joo, A. Lee, Y. Gan, Y. Yin, Chem. Commun. 49 (2013) 5135-5137.

[20] N. Nishiyama, T. Zheng, Y. Yamane, Y. Egashira, K. Ueyama, Carbon 43 (2005) 269274.

[21] X. Fang, J. Zang, X. Wang, M.-S. Zheng, N. Zheng, J. Mater. Chem. A 2 (2014) 61916197.

[22] J. Shen, Y. Hu, M. Shi, X. Lu, C. Qin, C. Li, M. Ye, Chem. Mater. 21 (2009) 35143520. 


\section{Figures and Captions}

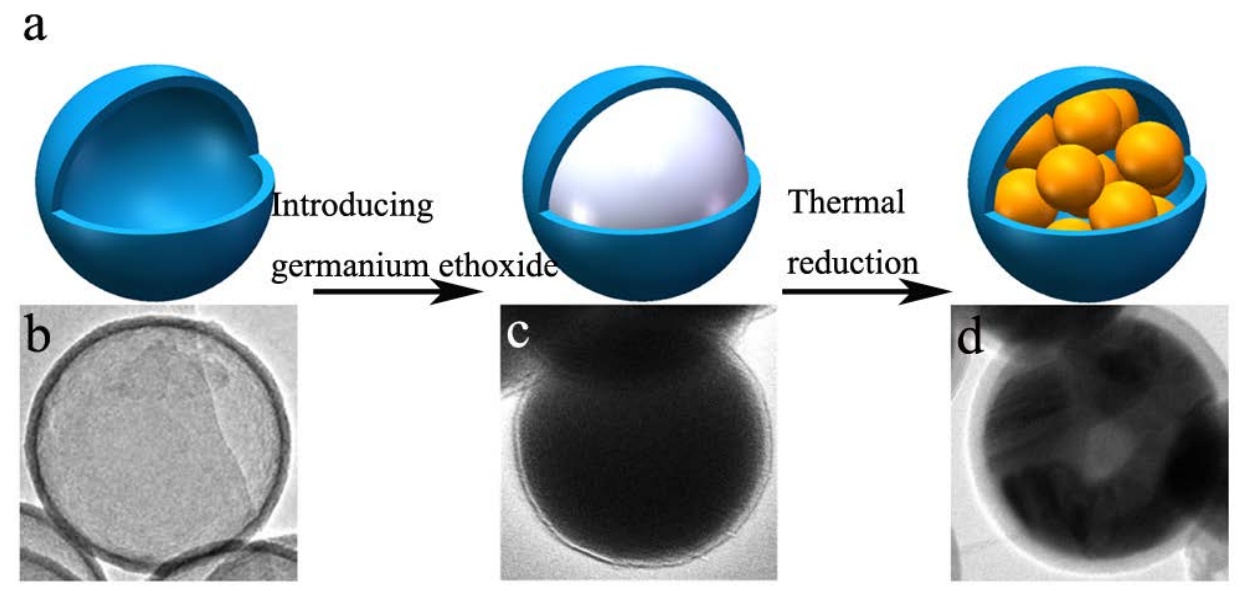

Figure 1. (a) Schematic illustration of the formation process to incorporate the germanium in the hollow carbon spheres. TEM images of (b) HCS, (c) GeO $\mathrm{G}_{2} @ \mathrm{HCS}$, and (d) Ge@HCS. 

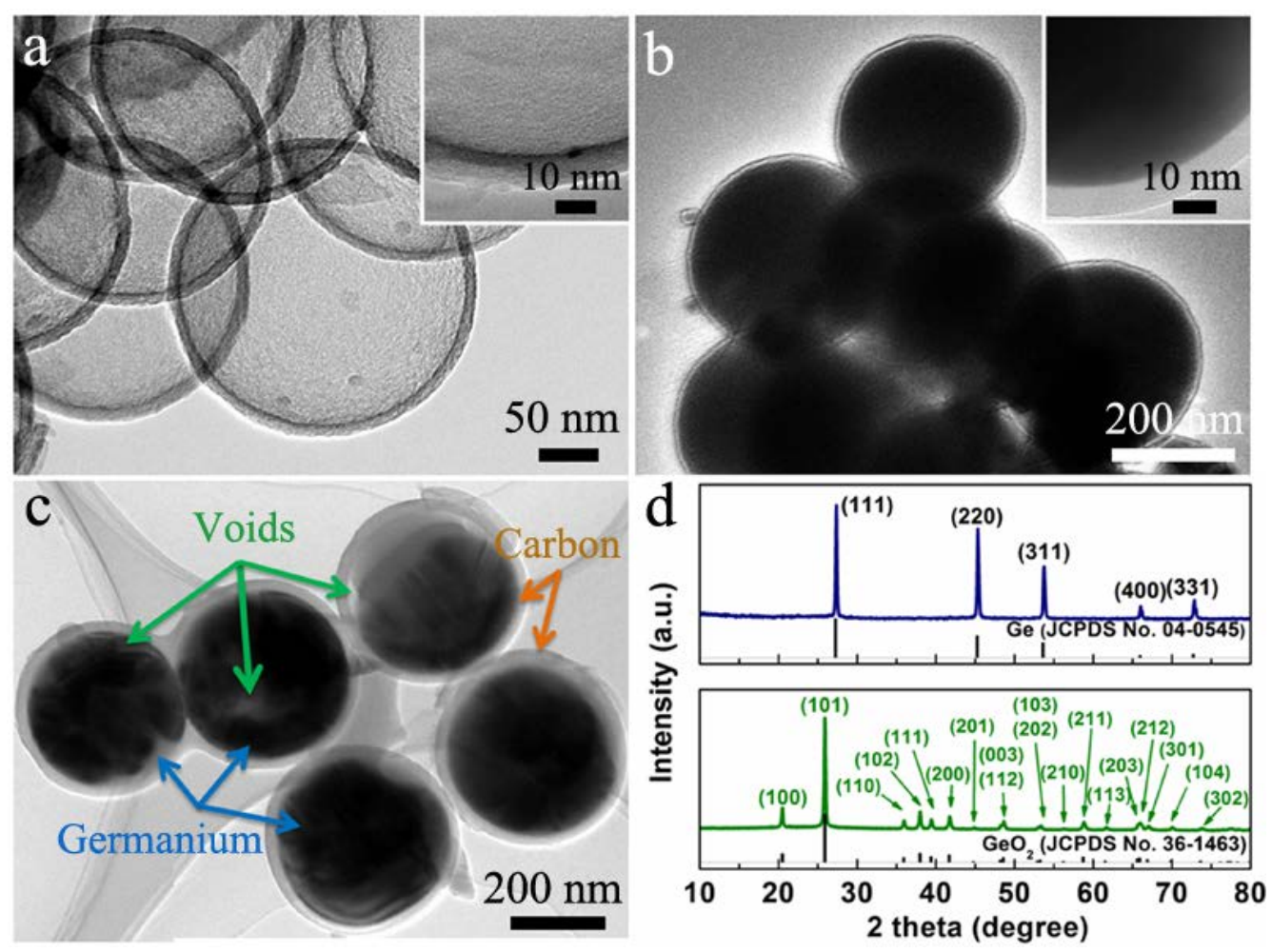

Figure 2. TEM images of (a) HCS, (b) $\mathrm{GeO}_{2} @ H C S$, and (c) Ge@HCS. (d) Powder X-ray diffraction patterns of the obtained $\mathrm{GeO}_{2} @ \mathrm{HCS}$ and Ge@HCS samples. 

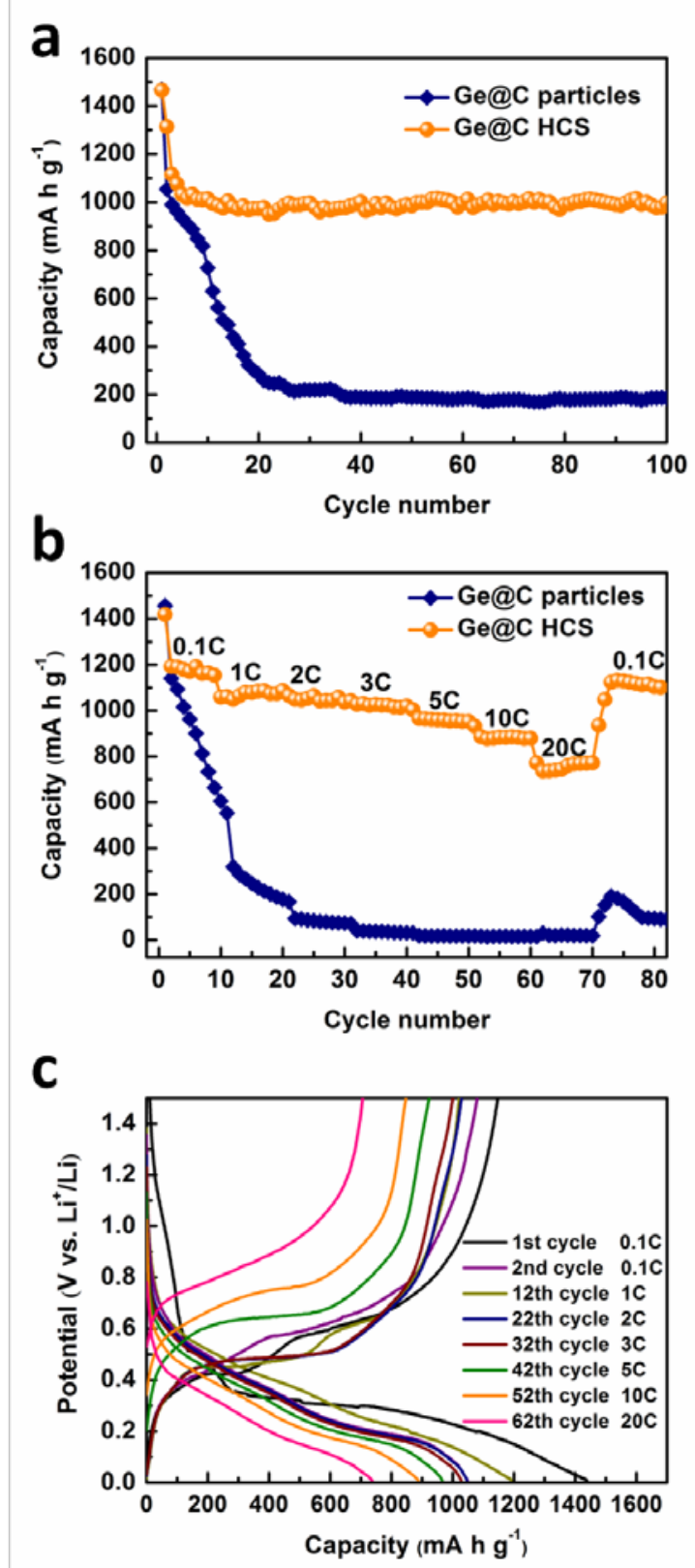

Figure 3. (a) Cycling performance of the Ge@C particles and the Ge@HCS sample at 0.4 C current rate over 100 cycles. (b) Comparison of rate capability of Ge@C particles and Ge@HCS sample at different current densities (charging rates from 0.1-20 C, with discharge at 0.2 C). (c) Galvanostatic charge-discharge profiles of the Ge@HCS composite at different current densities from 0.1-20 C (corresponding to (b)). 